$18 \mid 2014$

NOVECENTO... E DINTORNI

Da Torino a Parigi: Laura Malvano storica e critica d'arte

\title{
Nella Marchesini illustratrice di Cadetti di Kuprin nella traduzione di Ada Gobetti
}

Nella Marchesini illustratrice des Cadets de Kuprin dans la traduction italienne d'Ada Gobetti

Nella Marchesini Illustrator of The Cadets by Kuprin in Ada Gobetti's Italian Translation

Cesare Pianciola

\section{OpenEdition}

\section{Journals}

Edizione digitale

URL: http://journals.openedition.org/cei/1610

DOI: $10.4000 /$ cei. 1610

ISSN: 2260-779X

\section{Editore}

UGA Éditions/Université Grenoble Alpes

\section{Edizione cartacea}

Data di pubblicazione: 30 mars 2014

Paginazione: 85-94

ISBN: 978-2-84310-268-4

ISSN: 1770-9571 


\title{
NELLA MARCHESINI ILLUSTRATRICE DI CADETTI \\ DI KUPRIN NELLA TRADUZIONE DI ADA GOBETTI
}

\author{
Cesare Pianciola
}

Ho sentito tante volte Laura parlare con affetto di Ada Prospero Marchesini Gobetti, della «zia Ada». Negli ultimi anni e finché la malattia glielo permise, quando veniva a Torino da Parigi, Laura non mancava di passare qualche ora al Centro studi Piero Gobetti, in via Fabro 6, a raccogliere materiali per le sue ricerche. Alcune di queste concernevano direttamente la cerchia gobettiana. Quando nel 200I ci furono le manifestazioni del centenario della nascita di Piero Gobetti, Laura partecipò alla mostra Gobetti e Casorati 19I8-1926, curata da Maria Mimita Lamberti all'Archivio di Stato di Torino, scrivendo un saggio per il catalogo ${ }^{\mathrm{I}}$.

Per ricordare Laura ho pensato a un piccolo contributo che legasse l'opera della madre Nella ad Ada Gobetti. Mi sono ricordato di un bel libro della biblioteca di Ada conservata al Centro studi, un'opera minore che poteva però essere un documento significativo dell'amicizia e della collaborazione tra di loro ${ }^{2}$. Ho immaginato che Laura, abituata a cercare i nessi tra i documenti della cultura figurativa e la storia della cultura in senso più ampio, avrebbe approvato questa scelta.

I. È nota l'amicizia che legò Piero Gobetti e Ada Prospero alle sorelle Marchesini: Maria (I896-I933, che collaborò a «Energie Nove» e pubblicò

I. L. Malvano, Il pittore e il critico: note sulla ricezione della prima monografia casoratiana, in Piero Gobetti e Felice Casorati I9I8-1926, catalogo a cura di Rosanna Maggio Serra, Milano, Ministero per i Beni e le Attività culturali - Electa, 200I, pp. 23-30. Ma si veda pure L. Malvano Bechelloni, Piero Gobetti critico d'arte: "dignità» e "buon costume» alle prese con l'arte "reggitrice di stato», in M. CASSAC (a cura di), Piero Gobetti et la culture des années 20. Actes du Colloque international, Nice 30 et 31 octobre 1998, Nice, Publication de la Faculté des Lettres Arts et Sciences Humaines, 1999-2000, pp. 215-238.

2. A. Kuprin, I Cadetti. Romanzo. Unica versione integrale e conforme al testo russo, con note di Ada Prospero. Illustrazioni di Nella Marchesini, Torino, Alberto Giani Editore, 1927, II2 pp., formato cm I9 x 25,5, rilegatura editoriale. 
il saggio Omero nelle Edizioni del Baretti nel 1926), Ada (detta Dadi, I899I948) e Nella (I9OI-I953) 3

Nella fu presentata da Gobetti a Felice Casorati nel 1920 e divenne una delle allieve più promettenti negli studi di via Mazzini 52 e di via Galliari 33 .

Al primo piano [di via Galliari] ecco la Scuola di Felice Casorati — ha scritto Enrico Paulucci in una testimonianza del 1988 - un seguito di stanze dove in un silenzio severo e un po' allucinante, in piedi ai cavalletti tra nature morte posate su seggiole e sgabelli, maschere di gesso, una modella, un manichino, ecco gli allievi in lunghi grembiuloni grigi: Mario Bionda e Sergio Bonfantini, la trasgressiva Tina Mennyey, la materna Ida Donati, Paola Levi Montalcini, la bella Giorgina Lattes, Lalla Romano; ma su tutto, presenza gentile e quasi astratta, bionda, occhi chiari, viso pulito [...], ecco Nella Marchesini: la prediletta, quasi figura scesa da una tavola senese, da un frammento di affresco di Piero, il più casoratiano dei maestri del mondo casoratiano di allora ${ }^{4}$.

Si dovrebbe ricordare, tra altri allievi tralasciati da Paulucci, anche Silvio Avondo, con il quale Nella espose per la prima volta alcuni disegni — insieme al comune maestro - alla Galleria centrale di Torino nel I92I5.

Il nome delle sorelle Marchesini ricorre molte volte nella corrispondenza tra Piero e $\mathrm{Ada}^{6}$, dove sono ricordate sempre come amiche carissime a cui Piero faceva leggere i suoi lavori. Per esempio, a proposito dell'articolo Felice Casorati, comparso su "Poesia ed Arte» nel I920, Piero scriveva alla fidanzata: «Ho finito finalmente l'articolo su Casorati che riesce insomma a piacermi. Vi risolvo per me dei problemi che molto mi assillavano e mi par proprio di penetrare nell'intimo del processo creativo. È piaciuto moltissimo anche alle Marchesini e a Carlo Levi che l'hanno letto» ${ }^{7}$.

Dopo la morte di Piero, e il matrimonio di Nella con il pittore Ugo Malvano nel 1930, Ada continuò a frequentare intensamente la famiglia Marchesini e nel giugno 1937 sposò il fratello di Maria, Dadi e Nella, Ettore Marchesini. Anche Ettore aveva inclinazioni artistiche e nel 1940

3. Cfr. la scheda biografica di E. Alessandrone Perona su Maria Marchesini e le sorelle in appendice a P. Gobetti, Carteggio I9I8-I922, Torino, Einaudi, 2003, pp. 496-497. Su Nella Marchesini, oltre ai testi indicati nelle note successive, si veda il bel saggio di G. Romano, Percorso di Nella Marchesini, nel catalogo della mostra Nella Marchesini. Una storia familiare, Torino, Galleria del Ponte, 2012.

4. L. Riccio (a cura di), Nella Marchesini, Torino, Le Immagini, Studio d'arte contemporanea, 1989, p. 20.

5. Dettagliate notizie sulla scuola nella tesi di Veronica Cavallaro, La scuola di Casorati, Corso di laurea specialistica in storia del patrimonio archeologico e storico-artistico, Università di Torino, a. a. 20II-20I2, relatrice: prof.ssa Federica Rovati.

6. Cfr. P. e A. Gobetti, Nella tua breve esistenza. Lettere I9I8-1926, a cura di E. Alessandrone Perona, Torino, Einaudi, I991, ad indicem.

7. Ivi, p. 367. 
illustrò "con disegni naïf ma non troppo» ${ }^{8}$ il fortunato libro di Ada per i ragazzi La storia del gallo Sebastiano ovvero il tredicesimo uovo pubblicato nel 1940 presso Garzanti con lo pseudonimo di Margutte, poi ristampato dopo la guerra col vero nome dell'autrice ancora da Garzanti e successivamente in numerose edizioni da Einaudi ${ }^{9}$.

Molti sono dunque i fili che legano la cultura artistica della famiglia Marchesini agli interessi di Piero e Ada Gobetti. Cercheremo di metterne in luce alcuni partendo dal lavoro comune intorno a I cadetti di Aleksandr Ivanovič Kuprin.

2. Il volume uscì presso l'editore-tipografo torinese Alberto Giani nel I927 — l'anno successivo alla morte di Piero a Parigi (fig. I). Alcuni disegni originali per questa edizione sono conservati nell'Archivio Ugo Malvano e Nella Marchesini, e furono esposti nel $1994^{\mathrm{IO}}$.

Nel nutrito catalogo di Alberto Giani, nel quale si trovano libri molto vari, avevano spicco i romanzi storici di ambiente piemontese e sabaudo di Luigi Gramegna (I846-I928). Ma Giani pubblicò anche nel I924 la rivista letteraria "Contemporaneo", tra i cui redattori troviamo il giovane Marziano Bernardi, che sarà poi uno stimato critico d'arte. Giani fece inoltre uscire dal marzo 1922 al 1927 il quindicinale per ragazzi «Cuor d'oro", scritto da firme importanti dell'epoca (tra cui Giovanni Bertinetti, Renzo Pezzani, Angiolo Silvio Novaro), e illustrato da giovani e meno giovani artisti torinesi (tra i quali il grande Attilio Mussino, divenuto celebre per il Pinocchio uscito da Bemporad nel I9II) e di altre città ${ }^{\text {II }}$. Alcuni racconti uscivano sia sulla rivista che in volume illustrato: è il caso dei Cadetti di Alessandro (Alexandr) Kuprin, che uscì anche a puntate sulla rivista nel 1927, ultimo anno di vita del periodico ${ }^{\text {I2 }}$.

Un altro volume di letteratura russa pubblicato da Alberto Giani nel I924 era stato Colloquio notturno di Leonida Andreief (Leonid Andreev), nella traduzione di Bella Markmann e Carlo Staffetti.

Andreev e Kuprin erano autori cui Piero e Ada dedicarono le loro fatiche di giovanissimi traduttori dal russo, poco dopo l'inizio dello studio

8. G. Fofi, Tra memorialistica e narrativa, «Mezzosecolo. Materiali di ricerca storica», 7, Annali 1987-I989, pp. 314-315. Il numero è interamente dedicato alla figura e all'opera di Ada Prospero Marchesini Gobetti.

9. Sui libri per ragazzi scritti da Ada vedi ora: S. Gallino, Ada Gobetti civitas magna et opulenta opperuit $m e$, Torino, Beppe Grande, 2oII. Il libro ha in prefazione un'accurata nota biografica di Pietro Polito.

IO. Cfr. Nella Marchesini. Disegni I920-I952, Torino, Galleria Le Immagini, I994; quattro disegni per I cadetti di Kuprin sono riprodotti nel catalogo, pp. I9-2I.

II. Sull'attività di Alberto Giani e sulle sue riviste vedi A. D'Orsi, La cultura a Torino tra le due guerre, Torino, Einaudi, 2000, pp. 9I-93.

I2. Precisamente nei sei fascicoli che uscirono dal giugno al dicembre 1927, anno VI della rivista. 
acuto, disperato odio per l'insegnamento e per quell'orticello che era chiamato a coltivare.

Perciò i convittori approfittavano impunemente dei mi, nuti in cui il pesante sonno dell'ubbriachezza s'impadroniva della testa malata di Ivàn Arcbìpovic. Subito qualcuno dei "deboli, era messo di sentinella presso la porta, i più intraprendenti salivano

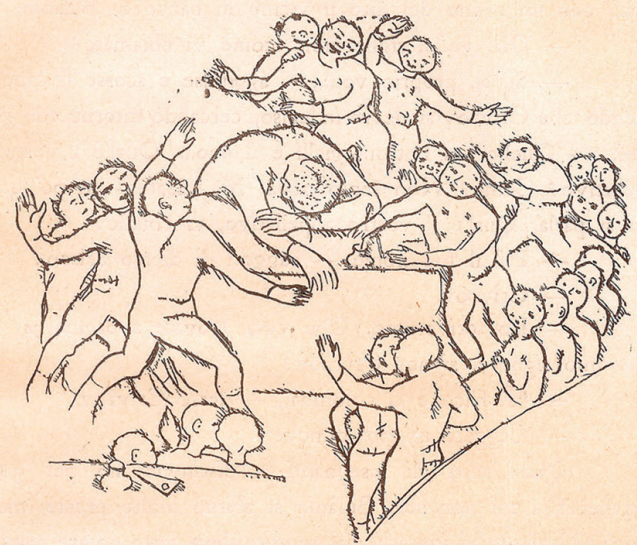

sulla cattedra, cancellavano dal registro $i$ voti e ne mettevano altri secondo il proprio giudizio, estraevano dalla tasca del professore l'orologio e lo guardavano, gli sporcavano col gesso la schiena. Perô, dobbiam dire a loro onore, che non appena la sentinella, udendo di lontano i pesanti passi dell'ispettore, gettava l'allarme: "Sst!... Tòlcac vienel...., immediatamente decine di mani servi, zievoli, sebben poco cerimoniose, si accingevano a scuotere Ivàn Archipovic.

Fig. I. - Pagina 2I de I cadetti.

\section{di questa lingua nel 1918 sotto la guida di Rachele Gutmann Polledro ${ }^{13}$. A Maria, Ada e Nella Marchesini «con l'affetto che lega il comune fervore di lavoro» era dedicata la loro traduzione, comparsa nel 1920 nella Biblioteca}

13. Un elenco molto accurato delle traduzioni di Ada Prospero, redatto da Sergio Caprioglio, si trova nel fascicolo di «Mezzosecolo» dedicato a Ada sopra citato e nel volumetto L'anno di Ada. Per il trentesimo anniversario della scomparsa di Ada Prospero Marchesini Gobetti, Torino, Centro studi Piero Gobetti, I998. Quanto alla letteratura russa, dopo la morte di Piero, oltre al volume di Kuprin I cadetti, Ada pubblicò nello stesso anno (1927) presso la casa editrice torinese Slavia, diretta da Alfredo Polledro, una raccolta di racconti di Tolstoj, tra cui il celebre La morte di Ivàn Iljic (il libro ebbe una terza edizione riveduta nel I933). Dopo il '27 Ada tradusse libri dal francese e soprattutto dall'inglese. 
universale Sonzogno, di Figlio dell'uomo e altre novelle di Andreev, un volumetto che nelle prime trenta pagine conteneva uno studio critico di Piero.

Di Kuprin (trascritto all'epoca anche come A. I. Cuprin) Piero e Ada avevano tradotto nello stesso periodo la raccolta di novelle Allez! ${ }^{I 4}$, con una presentazione introduttiva di Petr Pilschi e una Nota critica di Piero Gobetti datata novembre I920.

In questa breve appendice Gobetti scriveva che, se Andreev esprimeva il doloroso isolamento dello scrittore di fronte alla natura e alla storia, in Kuprin c'era un'accettazione della vita «nella sua evidenza e sicurezza, anche quando la sicurezza è soltanto convenzionalità — e l'evidenza espressivamente si fa generica». In un articolo più lungo e complesso sullo scrittore russo, pubblicato su «L'Ora» di Palermo il Io novembre 1923, Gobetti ribadiva che Kuprin, lontano dal realismo di Gogol, «si limita a lavorare secondo i più facili schemi veristici, con una sicurezza spaventosamente feconda», condita da motivi sentimentali di nostalgia. E aggiungeva: «Da questa nostalgia generica, che quasi esclude le sfumature e le finezze, nasce Cadetti, una delle sue opere più discusse, l'autobiografia di Cuprin fanciullo con descrizioni minuziose e romantiche della vita di collegio in Russia, delle sue monotonie, della sua esasperante tristezza» ${ }^{15}$.

Più positivo il giudizio su Kuprin di altri specialisti italiani di letteratura russa di quell'epoca, come Ettore Lo Gatto, che apprezzò le traduzioni di Piero e Ada e fece collaborare Piero alla sua rivista "Russia» ${ }^{16}$.

Ma vediamo il contenuto dei Cadetti, il breve romanzo in parte autobiografico che fu scritto da Kuprin a ventotto anni nel i898. Era stato pubblicato nel 1900 sulla rivista di Kiev "Zhizn' i iskusstvo» [La vita e l'arte], col titolo All'inizio. Schizzi di vita quotidiana al ginnasio militare e ripubblicato nel 1903, con altro titolo, nel primo volume dei racconti editi dalla casa editrice Znanie [La conoscenza] di Maksim Gorkij. Una versione ancora modificata uscì infine con il titolo definitivo I cadetti nel I906 sulla rivista «Niva».

Il racconto è ambientato in un collegio militare, dove il protagonista — il piccolo Bulànin — entra all'inizio accompagnato dalla madre che lo lascia, affettuosa e trepidante, raccomandandolo all'ufficiale che accoglie

I4. A. I. Cuprin, Allez!, tradotto direttamente dal russo da Piero Gobetti e Ada Prospero, «Il libro per tutti», $\mathrm{n}^{\circ}$ 6, Roma, La Voce, s. d. La traduzione, avverte una nota a p. I09, fu fatta nel I919. Nel i9r9 usciva anche la raccolta Il delirio, trad. di S. Morosova, Napoli, L'Editrice italiana, segno del successo in Italia dello scrittore russo.

I5. Cito da P. Gobetti, Paradosso dello spirito russo e altri scritti sulla letteratura russa, Torino, Einaudi, I976, pp. Iо8 е піо.

I6. Di E. Lo Gatto si veda soprattutto l'introduzione a A. I. Kuprin, Romanzi e racconti, Roma, De Carlo, I945, pp. 9-I6. 
i nuovi arrivati. Nel collegio i piccoli sono sottoposti ad ogni genere di angheria da parte dei ragazzi delle classi superiori e a poco a poco vi si rivelano i tratti di una «istituzione totale» in cui vige la sopraffazione e la legge del più forte, con una gerarchia di oppressori/oppressi di stampo carcerario. A un certo punto Kuprin interrompe il racconto, bozzettistico ma efficace, delle disavventure di Bulànin, per tracciare, nel quinto e sesto capitolo, una descrizione «esterna» e quasi sociologica del collegio, delle sue categorie di semi-reclusi, alcuni dei quali non hanno neppure una famiglia che li accolga nei fine-settimana. Il mondo dei ragazzi è parallelo e del tutto refrattario e impermeabile a quello degli adulti che li dovrebbero educare. Gli insegnanti sono una galleria di inetti e ubriaconi, con tic e stramberie oggetto della derisione e degli scherzi dei collegiali, i quali però non mancano di coprirli in occasione di ispezioni, mostrando una solidarietà tra oppressi che travalica le differenze di ruoli. È un ambiente in cui il piccolo alter ego del narratore diventa semischiavo, per un debito non pagato, di Grùzov, un ragazzo di quindici anni «con un giallo viso sospettoso di prigioniero", motivo per cui Bulànin si incanaglisce, trascura lo studio ed è condannato dal direttore ad una infamante fustigazione che gli apre una ferita nell'anima che lascerà i suoi segni nella vita successiva.

Benché il racconto sia intessuto anche di episodi divertenti e giocosi, scorre a tratti nel romanzo una corrente drammatica, con intenti di denuncia sociale, come in altri racconti di Kuprin (per esempio in uno dei più famosi, Il duello, che, quando comparve nel 1905, venne accolto da una parte della critica come una denuncia e una satira della vita militare).

3. Forse perché il pubblico cui si rivolgeva l'editore italiano era quello degli adolescenti - e proprio la scelta di farne un libro illustrato ne accentuava tale destinazione - le illustrazioni di Nella Marchesini non mettono in evidenza gli aspetti grotteschi e crudeli del romanzo e ne sottolineano invece il lato piacevole e favolistico, introducendo anche in molte vignette - e autonomamente rispetto al racconto che illustra — un sole con occhi, naso e bocca che fa da pendant alle teste rotonde dei cadetti e partecipa, ridendo o piangendo (fig. 2 ), alle vicende dei ragazzi. Non è un motivo del tutto marginale ed estrinseco perché ritorna nelle due altre prove di illustrazioni per ragazzi, rimaste inedite, che sono conservate tra le carte di Ada Prospero nell'Archivio del Centro studi Piero Gobetti di Torino ${ }^{17}$. La prima consiste in I9 fogli di disegni a china, alcuni dei quali acquerellati,

17. Centro studi Piero Gobetti, Fondo Ada Prospero Gobetti, serie 13, III, f 289. Il fondo è stato riordinato da Alessia Pedio. 
oltre a un bozzetto a tempera per la copertina. Nella li eseguì per il libro di Ada Prospero, Cinque bambini e tre mondi, pubblicato nel 1952, ma con tavole e disegni di un altro illustratore ${ }^{18}$. La seconda serie consiste in sei disegni a china che illustrano un breve racconto manoscritto della stessa Nella Marchesini, La gran festa di mezzo maggio. Questi fogli inediti si ricollegano per vari aspetti stilistici ai disegni per il libro di Kuprin, alcuni dei quali non furono utilizzati per il volume ${ }^{19}$.

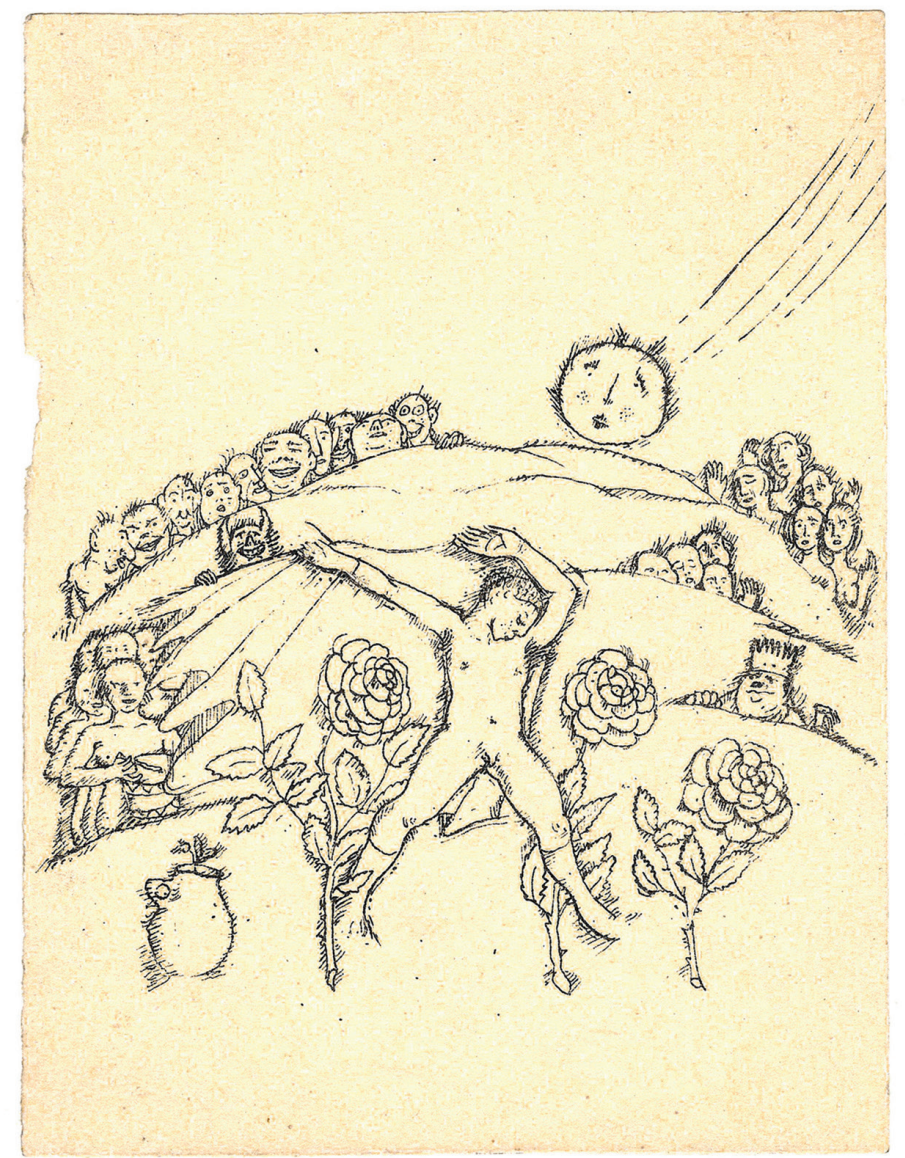

Fig. 2. - Un disegno originale per I cadetti non pubblicato nel libro.

18. A. Prospero, Cinque bambini e tre mondi, Torino, S.A.S. (Società Apostolato Stampa), I952.

19. Nell'Archivio Ugo Malvano e Nella Marchesini sono conservati: un disegno corrispondente alla figura di p. 8 del libro; uno studio per la stessa figura; altri 3 disegni che presentano qualche piccola variante rispetto a quelli pubblicati alle pp. 2, 6 e io del volume; 3 disegni che non sono stati inseriti nel libro a stampa. Devo la segnalazione ad Anna Malvano, che ringrazio. 
Un'analisi delle illustrazioni di Nella può essere condotta secondo due direzioni: possono essere collocate in riferimento alla sua pittura e in particolare alla sua attività di disegnatrice, che fu sempre intensa; e possono essere poste nel contesto degli illustratori italiani dei libri per l'infanzia ${ }^{20}$ nella seconda metà degli anni Venti.

Nelle illustrazioni di Nella le figure sono scabre, essenziali, tracciate con un sottile tratto di penna che evita ombre e chiaroscuri. Anche il tratteggio che compare in alcune vignette non corrisponde perlopiù ad ombre ma serve ad evocare il movimento. Certo si tratta di un lavoro minore, ma non alieno da quello che è il filo conduttore di quanto stava cercando come pittrice: «[...] la magrezza e la purezza "funzionale", smarrita tanto dal naturalismo descrittivo o allegorico quanto dall'astrazione decorativa. [...] per lei l'immagine dev'essere semplice e senza ornamento, primitiva nella nudità dell'apparire: una primitività raggiunta con lunga ricerca» ${ }^{2 \mathrm{r}}$.

Il disegno fu per Nella Marchesini sempre un'attività fondamentale tanto quanto la pittura, la quale del resto trovava nel disegno la base indispensabile ${ }^{22}$ : «Penso proprio - scriveva intorno al ' 48 Nella a Lia Rondelli — che la pittura poggia assolutamente sul disegno, è indispensabile come l'ossatura che se non c'è ben connessa il corpo cade giù». Nella fece moltissimi disegni che «stesi febbrilmente su pezzi di cartaccia ordinaria e casuale (ne ha lasciati centinaia e centinaia), non sono esercizi, non sono calligrafia, non saggi diligenti, curati e definitivi, non mai decorazione, sono parole del suo linguaggio di pittore [...]», come disse Casorati presentando nel 1956 la personale postuma della ex-allieva.

Quanto al confronto con gli illustratori del tempo e dell'ambiente torinese in cui Nella operava, quello più immediato è con gli artisti del quindicinale "Cuor d'oro». Alcuni (Carlo Nicco, che fu anche direttore artistico del periodico, Massimo Quaglino, Teonesto Deabate, Giulio Da Milano) si muovono tra modalità tradizionalmente descrittive e un disegno più libero e sintetico che traduce abilmente le acquisizioni delle avanguardie novecentesche (in questo eccelle soprattutto l'umbro-romano Mario Pompei). Attilio Mussino poi rielaborava con maestria gli stilemi del Liberty e dell'Art Déco. Le illustrazioni di Nella Marchesini risultano

20. Per un quadro complessivo cfr. A. Faeti, Guardare le figure. Gli illustratori italiani dei libri per l'infanzia, Torino, Einaudi, I972. Il volume è stato riedito con una nuova introduzione dell'autore nel $201 \mathrm{I}$ da Donzelli. Ma si veda anche il bel saggio di P. Vagliani, Figure d'infanzia, nel catalogo Infanzie. Il bambino nell'arte tra '80o e 'goo, Regione Piemonte, Torino, Edizioni Ages Arti Grafiche, 200I, pp. 20I-2I3.

2I. P. Mantovani, Dialogo fra due artisti compagni di vita, nel catalogo della mostra Ugo Malvano e Nella Marchesini. Un incontro di vita e d'arte, Rivarolo Canavese (To), 2008, p. 7.

22. Cfr. P. Mantovani, Il disegno di N. Marchesini, Catalogo della mostra di disegni a cura di Anna Malvano, con la collaborazione di Emanuela Genesio, Torino, Galleria del Ponte, 2007. Nel catalogo si trovano le citazioni utilizzate: quella della lettera a L. Rondelli a p. 6; quella di F. Casorati a p. II. 
originali e appartate rispetto a questi artisti, e si muovono su un piano di "elegante stilizzazione grafica» ${ }^{23}$ vicina ad alcune incisioni di Casorati degli inizi del Novecento $^{24}$, ma anche al disegno del maestro per un invito al Teatro Gualino del 1925, conservato nell'Archivio Ugo Malvano e Nella Marchesini (fig. 3). Anche Casorati aveva illustrato libri per ragazzi.
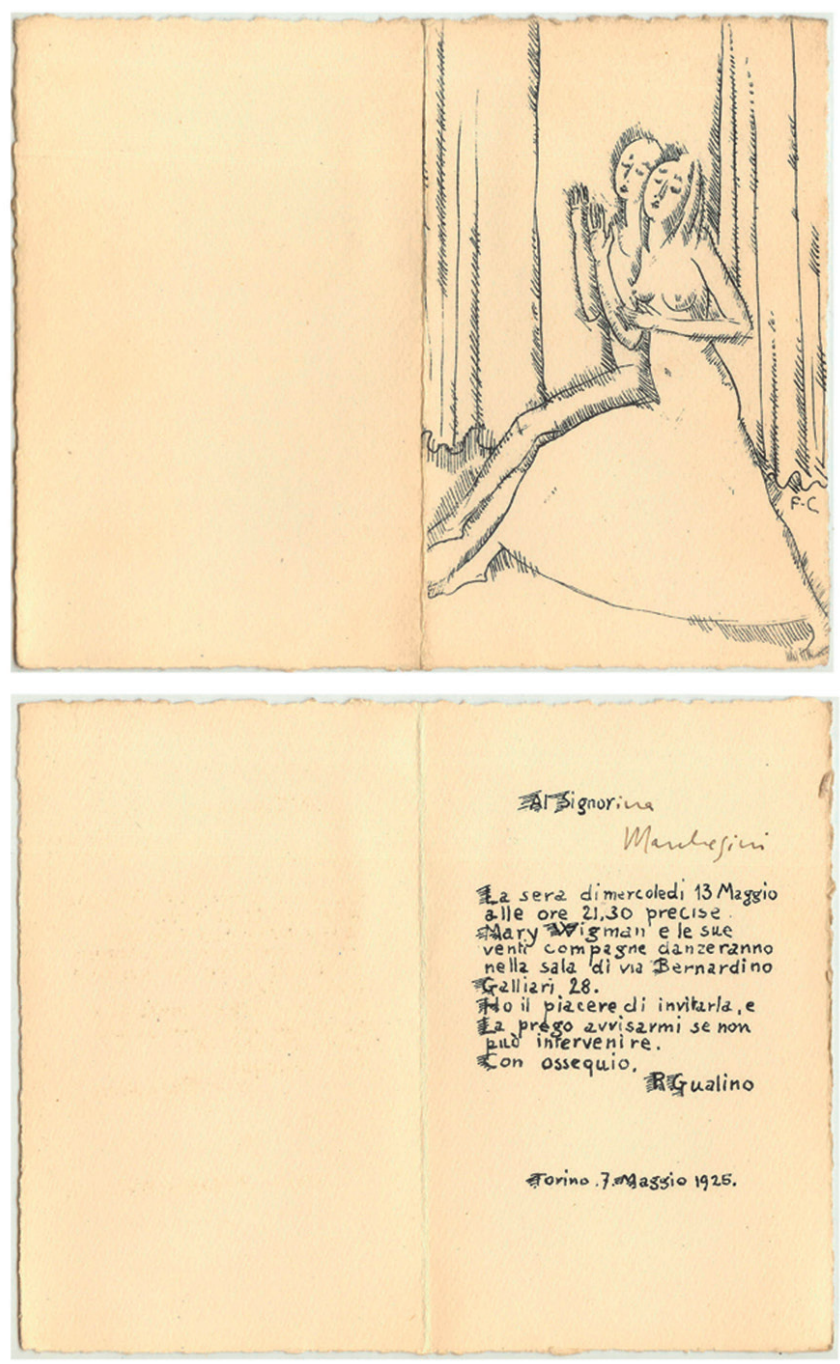

Fig. 3. - Felice Casorati, Invito del 1925 per il Teatro Gualino.

23. F. Poli, I disegni di Nella Marchesini, in Nella Marchesini. Disegni I920-I952, Torino, Le Immagini, Studio d'arte contemporanea, 1994, p. 9.

24. Cfr. ad esempio Donna (1908); Dialogo (1909); Allegoria (1910) in L. Carluccio e M. Rosci, Felice Casorati: Incisioni, sculture e disegni, scenografie, Padova, Franco Muizzio, 1985, pp. I7 sgg. 
Ricordiamo le belle illustrazioni in bianco e nero, un poco "metafisiche», che il pittore fece per un libro di Mario Buzzichini pubblicato da Alpes qualche anno prima ${ }^{25}$. Forse a queste si riferiva Piero Gobetti nella recensione alla mostra di grafica alla Galleria centrale d'arte nel I92I: «È rappresentata anche ampiamente la sua opera di illustratore di libri per fanciulli: nel genere che non vuol arte ma delicata letteratura, il Casorati ha raggiunto una finezza tutta sua ${ }^{26}$.

Nello stesso articolo, Gobetti sottolineava, a proposito di Nella Marchesini e di Silvio Avondo, "l'importanza d'un maestro che li ha liberamente dominati ed elevati, senz'alcuna meccanica influenza, a superare le costrizioni scolastiche e a valersi del mestiere per esprimere se stessi» ${ }^{27}$.

L'originalità dell'allieva rispetto al maestro si accentuerà nella inclinazione "espressionista» che via via manifestò sempre più apertamente e della quale, sul piano grafico, una grandissima prova sono soprattutto i disegni del 1944 in margine a Le laude di Jacopone da Todi. Alcuni furono esposti a Torino nel 1994 e nel 2007 alla Galleria Le Immagini e alla Galleria del Ponte. Sono disegni molto lontani dalle delicate litografie eseguite nel 1946 per l'Aminta di Tasso ${ }^{28}$, che dimostrano nel giro di pochi anni la capacità dell'artista di praticare diversi registri espressivi, sollecitata dai testi che di volta in volta traduce in immagini. I commenti grafici a Jacopone dell'epoca della guerra, tracciati con un forte bianco e nero, teso e spezzato, sono lontani sia dalle illustrazioni per I cadetti, sia da molte di quelle del 195I-1952 per Cinque bambini e tre mondi, dove Nella riprende l'antica ispirazione delle figure dei Cadetti, ritenendola probabilmente più confacente a un libro per ragazzi. Ma in alcune tavole per il libro del 1952 troviamo anche quel drammatico senso di desolazione per i disastri della guerra che certo alimentò i suoi disegni per Jacopone.

Il periodo della prima collaborazione artistica con l'amica Ada Gobetti era ormai molto lontano. Il I7 agosto 1953 - un anno dopo la scomparsa del marito - Nella moriva a soli cinquantun anni.

25. M. Buzzichini, Giocherello. Romanzo, disegni di F. Casorati, Milano, Alpes, I92I.

26. P. Gobetti, Scritti sull'arte, a cura di M. De Benedictis, Torino, Nino Aragno, 200o, p. 72.

27. Ibidem.

28. T. TAsso, Aminta, edizione in quarto, tiratura di 175 esemplari numerati, con nove litografie originali di Nella Malvano Marchesini, Torino, Collezione del Bibliofilo, I946. 